PROCEEDINGS OF THE

AMERICAN MATHEMATICAL SOCIETY

Volume 134, Number 10, October 2006, Pages 2957-2966

S 0002-9939(06)08555-8

Article electronically published on May 1, 2006

\title{
A SELECTION THEOREM IN METRIC TREES
}

\author{
A. G. AKSOY AND M. A. KHAMSI
}

(Communicated by Jonathan M. Borwein)

\begin{abstract}
In this paper, we show that nonempty closed convex subsets of a metric tree enjoy many properties shared by convex subsets of Hilbert spaces and admissible subsets of hyperconvex spaces. Furthermore, we prove that a set-valued mapping $T^{*}$ of a metric tree $M$ with convex values has a selection $T: M \rightarrow M$ for which $d(T(x), T(y)) \leq d_{H}\left(T^{*}(x), T^{*}(y)\right)$ for each $x, y \in M$. Here by $d_{H}$ we mean the Hausdroff distance. Many applications of this result are given.
\end{abstract}

\section{INTRODUCTION}

The study of injective envelopes of metric spaces, also known as metric trees ( $\mathbb{R}$-trees or T-theory), has its motivation in many subdisciplines of mathematics as well as biology/medicine and computer science. Its relationship with biology and medicine stems from the construction of phylogenetic trees 24]. Concepts of "string matching" in computer science are closely related with the structure of metric trees [5]. In the definition of an ordinary tree all the edges are assumed to have the same length, and therefore the metric is not often stressed. A metric tree is a generalization of an ordinary tree which allows for different length edges, thus emphasizing the behavior of free actions on metric trees. (For more details see [22], 23].) Metric trees were first introduced by J. Tits [26] in 1977. A metric tree is a metric space $(M, d)$ such that for every $x, y$ in $M$ there is a unique arc between $x$ and $y$, and this arc is isometric to an interval in $\mathbb{R}$. For example, a connected graph without a loop is a metric tree. One basic property of metric trees is their one-dimensionality. Around the same time period, while studying t-RNA molecules of the E. Coli bacterium, M. Eigen raised several questions which led A. Dress [11, 12 to construct metric trees (named as T-theory). Metric trees also arise naturally in the study of group isometries of hyperbolic spaces. For metric properties of trees we refer to [10. Topological characterization of metric trees were explored in 20] and 21], where it was proved that for a separable metric space $(M, d)$ the following are equivalent:

1. $M$ admits an equivalent metric $\rho$ such that $(M, \rho)$ is a metric tree.

2. $M$ is locally arcwise connected and uniquely arcwise connected.

Throughout this paper, we will make use of the hypercconvexity of metric trees. First let us recall the definition of a hyperconvex metric space.

Received by the editors April 27, 2005.

2000 Mathematics Subject Classification. Primary 47H04, 47H10, 54H25, 47H09.

Key words and phrases. Metric trees, selection theorems, hyperconvex spaces.

(C)2006 American Mathematical Society 
Definition 1.1. A metric space $(M, d)$ is said to be hyperconvex if $\bigcap_{i \in I} B\left(x_{i} ; r_{i}\right) \neq$ $\emptyset$ for any collection $\left(B\left(x_{i} ; r_{i}\right)\right)_{i \in I}$ of closed balls in $M$ for which $d\left(x_{i}, x_{j}\right) \leq r_{i}+r_{j}$, for any $i, j \in I$.

This notion was first introduced by Aronszajn and Panitchpakdi in [3], where it is shown that a metric space is hyperconvex if and only if it is injective with respect to nonexpansive mappings. Later Isbell [13] showed that every metric space has an injective hull, therefore every metric space is isometric with a subspace of a minimal hyperconvex space. Hyperconvex metric spaces are complete and connected [16]. The simplest example of hyperconvex space is the set of real numbers $\mathbb{R}$ or a finitedimensional real Banach space endowed with the maximum norm. While Hilbert space $l^{2}$ fails to be hyperconvex, the spaces $L^{\infty}$ and $l^{\infty}$ are hyperconvex.

There is a close connection between hyperconvex spaces and metric trees. In 2], authors take the definition of a metric tree from A. Dress and show that every complete metric tree is hyperconvex. On the other hand, in [18] using the definition of an $\mathbb{R}$-tree given by J. Tits, it is shown that a metric space $M$ is a complete $\mathbb{R}$ tree if and only if $M$ is hyperconvex with unique metric segments. For more about hyperconvex spaces and fixed point theorems in hyperconvex spaces we refer to [15] and 16. In this paper we focus on external hyperconvexity and utilize this concept to obtain a selection theorem for a set-valued function on a metric tree.

\section{BASIC DEFINITIONS AND RESUlts}

We begin by describing the relevant notation. For a subset $C$ of a metric space $M$, we denote the $\epsilon$-neighborhood of $C$ by $N_{\epsilon}(C)$,

$$
N_{\epsilon}(C)=\{x \in M: \operatorname{dist}(x, C) \leq \epsilon\},
$$

where $\operatorname{dist}(x, C)=\inf \{d(x, c) ; c \in C\}$.

Definition 2.1. A subset $C$ of a metric space $M$ is said to be externally hyperconvex relative to $M$ if, given any family of points $\left\{x_{\alpha}\right\}_{\alpha \in \Gamma}$ in $M$ and a family of $\left\{r_{\alpha}\right\}_{\alpha \in \Gamma}$ of real numbers satisfying

$$
d\left(x_{\alpha}, x_{\beta}\right) \leq r_{\alpha}+r_{\beta} \text { and } \operatorname{dist}\left(x_{\alpha}, C\right) \leq r_{\alpha}
$$

for any $\alpha, \beta \in \Gamma$, then it follows that $\bigcap_{\alpha \in \Gamma} B\left(x_{\alpha} ; r_{\alpha}\right) \cap C \neq \emptyset$.

Note that, in the above definition, centers of the balls $\left\{x_{\alpha}\right\}$ are not necessarily taken in $C$. This concept was originally introduced by Aronszajn and Panitchpakti in their seminal paper [3], where they have shown that:

1. Any admissible subset of a hyperconvex space $M$ is externally hyperconvex relative to $M$.

2. The externally hyperconvex subsets of $M$ are proximinal (i.e., if $C$ is externally hyperconvex relative to $M$ and if $x \in M$, then there exists $c^{*} \in C$ such that $\left.d\left(x, c^{*}\right)=\operatorname{dist}(x, C)\right)$.

It is known that not every hyperconvex space is externally hyperconvex [17. Throughout this paper we use $\mathcal{B}(M)$ to denote the family of all nonempty bounded subsets of $M$. We will put the Hausdorff metric $d_{H}$ on $\mathcal{B}(M)$. As usual, the Hausdorff metric is defined as

$$
d_{H}(A, B)=\inf \left\{\epsilon>0: A \subset N_{\epsilon}(B) \text { and } B \subset N_{\epsilon}(A)\right\} .
$$

Let $T^{*}: M \rightarrow \mathcal{B}(M)$ be a set-valued function. A selection is a map $T: M \rightarrow M$ such that $T(x) \in T^{*}(x)$ for all $x$ in $M$. Selection theorems appear in a variety 
of mathematical problems (or many problems in mathematics can be rephrased as selection problems). Depending on the context, one usually considers selections with some additional properties such as "continuous", "measurable", "nonexpansive," etc. For example in 25, R. Sine has a selection theorem for nonexpansive maps $T^{*}: M \rightarrow \mathcal{A}(M)$, where $\mathcal{A}(M)$ is the family of admissible subsets of the hyperconvex space $M$. For more on selection, and Michael's selection theorems in particular, we refer to [6. Selection theorems are also utilized in problems related to the extension of operators. One such example is due to M. Zippin 27, where by using Michael's continuous-selection theorem he obtains an extension theorem concerning linear maps from subspaces of $c_{0}$ into $C(K)$, with $K$ being compact and Hausdorff. In [16, a selection theorem was proved for set-valued mapping of a hyperconvex metric space $M$ which takes values in the space of nonempty externally hyperconvex subsets $H$ (relative to $M$ ). Our purpose here is to prove a similar theorem in the context of metric trees.

Since a metric tree is a space in which there is only one path between two points $x$ and $y$, this would imply that if $z$ is a point between $x$ and $y$, by which we mean if $d(x, z)+d(z, y)=d(x, y)$, then we know that $z$ is actually on the path between $x$ and $y$. This will motivate the next concept of a metric interval.

Definition 2.2. Let $(M, d)$ be a metric space and let $x, y \in M$. An arc from $x$ to $y$ is the image of a topological embedding $\alpha:[a, b] \rightarrow M$ of a closed interval $[a, b]$ of $\mathbb{R}$ such that $\alpha(a)=x$ and $\alpha(b)=y$. A geodesic segment from $x$ to $y$ is the image of an isometric embedding $\alpha:[a, b] \rightarrow M$ such that $\alpha(a)=x$ and $\alpha(b)=y$. The geodesic segment will be called a metric segment and denoted by $[x, y]$ throughout this work.

Now we are ready to define a metric tree.

Definition 2.3. A metric tree is a nonempty metric space $M$ satisfying:

(a) Any two points of $x, y \in M$, where $x$ and $y$ are the endpoints of a metric segment $[x, y]$.

(b) If $x, y, z \in M$, then $[x, y] \cap[x, z]=[x, w]$ for some $w \in M$ (i.e., if we have two metric segments with a common endpoint, then their intersection is a metric segment).

(c) If $x, y, z \in M$ and $[x, y] \cap[y, z]=\{y\}$, then $[x, y] \cup[y, z]=[x, z]$ (i.e., if two metric segments intersect in a single point, then their union is a metric segment).

Next we give some basic properties of metric segments.

Lemma 2.1. Let $(M, d)$ be a metric space and $x, y \in M$, with $x \neq y$.

1. If $z \in[x, y]$, then $[x, z] \subset[x, y]$.

2. If $M$ is a metric tree, then for any $z \in M$, there is a unique $w \in[x, y]$ such that

$$
[x, z] \cap[y, z]=[w, z] .
$$

The proof is classical and may be found in $[8]$.

Definition 2.4. Let $M$ be a metric tree and $C \subset M$. We say $C$ is convex if for all $x, y \in C$ we have $[x, y] \subset C$.

Clearly, a metric tree $M$ and $\emptyset$ are convex. Also, any closed ball $B(a, r)=\{z \in$ $M: d(a, z) \leq r\}$ in a metric tree is also convex. To see this, take two arbitrary 
elements $x, y$ of $B(a, r)$ and let $z \in[x, y]$. From the above lemma, there exists a unique $w \in[x, y]$ such that

$$
[x, a] \cap[y, a]=[w, a] .
$$

Since $[x, y]=[x, w] \cup[w, y]$, we have $z \in[x, w]$ or $z \in[w, y]$. Without loss of generality, assume $z \in[x, w]$. Then

$$
\begin{aligned}
d(a, z) & \leq d(a, w)+d(w, z) \leq d(a, w)+d(w, z)+d(x, z) \\
& =d(a, w)+d(x, w)=d(a, x) \leq r,
\end{aligned}
$$

which implies $z \in B(a, r)$.

The following notations will be referenced throughout this paper. Let $(M, d)$ be a metric tree and let $A$ be a nonempty bounded subset of $M$. Set

$$
\operatorname{co}(A)=\bigcap\{B: B \text { is a closed ball and } A \subset B\} .
$$

The subset $A$ is called admissible if $c o(A)=A$ (i.e., $A$ is an intersection of closed balls). Let $\mathcal{A}(M)$ denote the collection of admissible subsets in $M$ and let $\mathcal{C}(M)$ denote the collection of all closed convex subsets of $M$. We set

$$
\operatorname{conv}(A)=\bigcap\{B: B \text { is a convex subset of } M \text { such that } A \subseteq B\} .
$$

Since closed balls are convex, we have $\mathcal{A}(M) \subset \mathcal{C}(M)$. Moreover, this is a proper inclusion. To see this, we need to make a few observations. First, if one has $x \neq y$ with $[x, y] \subset B(a, r)$ and $m$ is the midpoint of $[x, y]$, then

$$
B\left(m, \frac{d(x, y)}{2}\right) \subset B(a, r)
$$

which implies $B\left(m, \frac{d(x, y)}{2}\right) \subset c o([x, y])$. Indeed, considering $z \in B\left(m, \frac{d(x, y)}{2}\right)$, using the properties of metric trees, we have $d(a, w)+d(w, y)=d(a, y)$ or

$$
d(a, w)+d(w, m)+d(m, y)=d(a, y) \leq r,
$$

which implies that $d(a, w)+d(w, m) \leq r-d(x, y) / 2$. The triangle inequality will then imply

$$
d(a, z) \leq d(a, m)+d(m, z) \leq r-\frac{d(x, y)}{2}+\frac{d(x, y)}{2}
$$

and therefore $z \in B(a, r)$, and from the definition of $\operatorname{cov}([x, y])$ we also have $z \in$ $c o([x, y])$. Moreover closed unit balls in a metric tree may not be compact. Indeed, take a closed unit ball in $\mathbb{R}^{2}$ with a radial metric. Looking at $x, y \in B(0,1)$ with $x \neq y$, then the radial distance between $x$ and $y$ is 2 . Also any metric segment in $B(0,1)$ is contained in $c o([x, y])$, which implies that $c o([x, y])$ is not compact for this distance. But $[x, y]$ is compact and convex. This example was suggested to us by Kirk [19. For more examples, we suggest the wonderful paper by Bestvina [7.

The following result due to Baillon $[4$ is crucial in our investigation of $\mathcal{C}(M)$.

Theorem 2.1 (4]). Let $M$ be a bounded metric space and let $\left\{H_{\beta}\right\}_{\beta \in \Gamma}$ be a decreasing family of nonempty hyperconvex subsets of of $M$. Then $\bigcap_{\beta \in \Gamma} H_{\beta} \neq \emptyset$ and is hyperconvex. 


\section{THE NEAREST POINT MAP}

Let $C$ be a closed convex subset of a metric tree $M$. If for every point $x \in M$ there exists a nearest point in $C$ to $x$, and if this point is unique, we denote this point by $P_{C}(x)$, and call the mapping $P_{C}$ the metric projection from $M$ into $C$. In a Hilbert space, the metric projections on closed convex subsets are nonexpansive. In uniformly convex spaces, the metric projections are uniformly Lipschitzian. In fact, they are nonexpansive if and only if the space is Hilbert. In what follows we will show that these metric projections on closed convex subsets of metric trees are nonexpansive. This result is not known in hyperconvex metric spaces. Indeed, one can show that a nonexpansive retract into an admissible subset always exists which may not be in general the nearest point projection.

The existence of a nearest point relies on some kind of compactness which reduces to the reflexivity in the linear case.

Theorem 3.1. Let $M$ be a bounded metric tree. Then $\mathcal{C}(M)$ is compact (i.e., for any family $\left\{C_{\beta}\right\}_{\beta \in \Gamma}$ in $\mathcal{C}(M)$ such that $\bigcap_{\beta \in \Gamma_{f}} C_{\beta} \neq \emptyset$, where $\Gamma_{f}$ is any finite subset of $\Gamma$, we have

$$
\bigcap_{\beta \in \Gamma} C_{\beta} \neq \emptyset
$$

and is in $\mathcal{C}(M))$. Moreover $\mathcal{C}(M)$ is uniformly normal.

The compactness of $\mathcal{C}(M)$ is a direct consequence of Baillon's theorem. Indeed, convex subsets of metric trees are subtrees. Therefore they must be hyperconvex. The uniform normality of $\mathcal{C}(M)$ is also consequence of the hyperconvexity property. In this case, we have

$$
R(C)=\inf \left\{\sup _{x \in C} d(x, c) ; c \in C\right\}=\frac{1}{2} \operatorname{diam}(C)
$$

for any bounded nonempty closed convex subset $C$.

Next we discuss the proximinality of the closed convex subsets of a metric tree.

Lemma 3.1. If $C$ is a nonempty bounded convex subset of a metric tree $M$, then

1. For any $x \in M$ there exists $c_{x} \in C$ such that

$$
d(x, C)=\inf \{d(x, c) ; c \in C\}=d\left(x, c_{x}\right) .
$$

2. For any $x \in M$, the set $P_{C}(x)$ is a singleton (i.e., $P_{C}$ is single-valued). Moreover if $c \in C$ we have

$$
d\left(x, P_{C}(x)\right)+d\left(P_{C}(x), c\right)=d(x, c)
$$

(i.e., $P_{C}(x) \in[x, c]$ is the unique segment joining $x$ to $c$ ).

Proof. 1. Since $C$ is not empty, the number $d(x, C)$ is finite. Let $\varepsilon>0$; then

$$
C_{\varepsilon}=C \cap B(x, d(x, C)+\varepsilon)
$$

is a bounded nonempty closed convex subset of $C$. The compactness of $\mathcal{C}(M)$ implies that

$$
P_{C}(x)=\{c \in C ; d(x, c)=d(x, C)\}
$$

is not empty.

2. We know that $P_{C}(x)$ is not empty. Let $c_{x} \in P_{C}(x)$ and $c \in C$. Then, since $M$ is a metric tree, there exists $z \in M$ such that

$$
[c, x] \cap\left[c, c_{x}\right]=[c, z] .
$$


Because $C$ is convex, we have $z \in\left[c, c_{x}\right] \subset C$. We also have

$$
d(x, z)+d\left(z, c_{x}\right)=d\left(x, c_{x}\right)
$$

so

$$
d(x, C) \leq d(x, z) \leq d(x, z)+d\left(z, c_{x}\right)=d\left(x, c_{x}\right)=d(x, C),
$$

which obviously implies $d\left(z, c_{x}\right)=0$ or $z=c_{x}$. So $c_{x} \in[x, c]$, which yields what we want. Clearly this will imply that $P_{C}(x)$ is reduced to one point.

Next, we discuss another behavior of the retract $P_{C}$.

Lemma 3.2. Let $x, y \in M$. Set $c_{x}=P_{C}(x), c_{y}=P_{C}(y), r(x)=d(x, C)=d\left(x, c_{x}\right)$ and $r(y)=d(y, C)=d\left(y, c_{y}\right)$. We have either

$$
d\left(c_{x}, c_{y}\right)=d(x, y)-r(x)-r(y) \leq d(x, y)
$$

or $c_{x}=c_{y}$. In particular, $P_{C}$ is nonexpansive.

Proof. Since $M$ is a metric tree, there exists $z \in M$ such that $[y, x] \cap\left[y, c_{x}\right]=[y, z]$. Assume $z \in C$, since $z \in\left[x, c_{x}\right]$. Based on what we have shown above, we must have $z=c_{x}$. So $d\left(x, c_{x}\right)+d\left(c_{x}, y\right)=d(x, y)$. Since $d\left(c_{x}, c_{y}\right)+d\left(c_{y}, y\right)=d\left(c_{x}, y\right)$, we get

$$
d\left(x, c_{x}\right)+d\left(c_{x}, c_{y}\right)+d\left(c_{y}, y\right)=d(x, y)
$$

which implies $r(x)+d\left(c_{x}, c_{y}\right)+r(y)=d(x, y)$ or

$$
d\left(c_{x}, c_{y}\right)=d(x, y)-r(x)-r(y) .
$$

Assume that $z \notin C$, and consider $c_{z}=P_{C}(z)$. Since $z \in\left[c_{x}, x\right]$ we get $d\left(x, c_{x}\right)=$ $d(x, z)+d\left(z, c_{x}\right)$, and from the above properties we have $d\left(c_{x}, c_{z}\right)+d\left(c_{z}, z\right)=$ $d\left(c_{x}, z\right)$. So

which yields

$$
d(x, z)+d\left(z, c_{z}\right)+d\left(z, c_{x}\right)=d\left(x, c_{x}\right),
$$

$$
d\left(x, c_{z}\right) \leq d(x, z)+d\left(z, c_{z}\right) \leq d(x, z)+d\left(z, c_{z}\right)+d\left(z, c_{x}\right)=d\left(x, c_{x}\right) .
$$

By the definition of $P_{C}(x)$ we get $c_{z}=c_{x}$. A similar argument will lead to $c_{z}=$ $c_{x}=c_{y}$.

The fact that the nearest point projection onto convex subsets of metric trees is nonexpansive also follows from the fact that this is true in the more general setting of $\mathrm{CAT}(0)$ spaces (see p. 177 of [9]).

The next theorem is crucial for our main result. It shows that convex subsets are little more than just hyperconvex. They are externally hyperconvex, which was previously unknown.

Theorem 3.2. If $C$ is a nonempty convex subset of a metric tree $M$, then $C$ is externally hyperconvex with respect to $M$.

In other words, for any $\left(x_{\alpha}\right)_{\alpha \in \Gamma}$ in $M$ and $\left(r_{\alpha}\right)_{\alpha \in \Gamma}$ positive numbers such that

$$
d\left(x_{\alpha}, x_{\beta}\right) \leq r_{\alpha}+r_{\beta}
$$

and

$$
d\left(x_{\alpha}, C\right) \leq r_{\alpha}
$$

for any $\alpha, \beta \in \Gamma$, then

$$
C \cap\left(\bigcap_{\alpha \in \Gamma} B\left(x_{\alpha}, r_{\alpha}\right)\right) \neq \emptyset
$$


Proof. First set

$$
P_{C}\left(x_{\alpha}\right)=c_{\alpha}
$$

and

$$
r\left(x_{\alpha}\right)=d\left(x_{\alpha}, c_{\alpha}\right)=d\left(x_{\alpha}, C\right)
$$

for any $\alpha \in \Gamma$. Then for any $\alpha, \beta \in \Gamma$ we have either

$$
d\left(c_{\alpha}, c_{\beta}\right)=d(x, y)-r\left(x_{\alpha}\right)-r\left(x_{\beta}\right) \leq d(x, y)
$$

or $c_{\alpha}=c_{\beta}$. Note that our assumption implies

$$
r\left(x_{\alpha}\right)=d\left(x_{\alpha}, c_{\alpha}\right)=d\left(x_{\alpha}, C\right) \leq r_{\alpha} .
$$

In both cases we have

$$
d\left(c_{\alpha}, c_{\beta}\right) \leq r_{\alpha}+r_{\beta}-r\left(x_{\alpha}\right)-r\left(x_{\beta}\right) .
$$

Since $C$ is hyperconvex we have

$$
C^{*}=C \cap\left(\bigcap_{\alpha \in \Gamma} B\left(c_{\alpha}, r_{\alpha}-r\left(x_{\alpha}\right)\right)\right) \neq \emptyset .
$$

Let $c \in C^{*}$. Then we have $c \in C$ and

$$
d\left(c, c_{\alpha}\right) \leq r_{\alpha}-r\left(x_{\alpha}\right)
$$

but

$$
d\left(x_{\alpha}, c\right)=d\left(x_{\alpha}, c_{\alpha}\right)+d\left(c_{\alpha}, c\right)=r\left(x_{\alpha}\right)+d\left(c, c_{\alpha}\right) \leq r_{\alpha} .
$$

In other words, we have

$$
c \in C \cap\left(\bigcap_{\alpha \in \Gamma} B\left(x_{\alpha}, r_{\alpha}\right)\right),
$$

which completes the proof of our theorem.

In the next section, we will discuss how using the above results shows the existence of selections to multivalued maps.

\section{MAin RESUlts}

The selection theorem stated here is similar to the main result of [16]. Throughout this section $\mathcal{C}(M)$ denotes the family of all nonempty bounded closed convex subsets of a metric tree $M$.

Theorem 4.1. Let $M$ be a metric tree and $T^{*}: M \rightarrow \mathcal{C}(M)$. Then there exists a mapping $T: M \rightarrow M$ for which $T(x) \in T^{*}(x)$ for each $x \in M$ and for which

$$
d(T(x), T(y)) \leq d_{H}\left(T^{*}(x), T^{*}(y)\right)
$$

for each $x, y \in M$.

Proof. Let $\mathcal{F}$ denote the collection of all pairs $(A, T)$ such that

$$
\left\{\begin{array}{l}
T: A \rightarrow M, \\
T(a) \in T^{*}(a), \\
d(T(x), T(y)) \leq d_{H}\left(T^{*}(x), T^{*}(y)\right)
\end{array}\right.
$$

for all $a \in A$, and $x, y \in M$. Since $(\{x\}, T) \in \mathcal{F}$ for any choice of $x \in M$ and $T(x) \in T^{*}(x)$, we have $\mathcal{F} \neq \emptyset$. Define an order relation on $\mathcal{F}$ by setting

$$
\left(A_{1}, T_{1}\right) \preceq\left(A_{2}, T_{2}\right) \Leftrightarrow A_{1} \subset A_{2} \text { and }\left.T_{2}\right|_{A_{1}}=T_{1} .
$$


Let $\left\{\left(A_{\alpha}, T_{\alpha}\right)\right\}$ be an increasing chain in $(\mathcal{F}, \preceq)$. Then it follows that $\left(\bigcup_{\alpha} A_{\alpha}, T\right) \in$ $\mathcal{F}$, where $T \mid A_{\alpha}=T_{\alpha}$. This element is obviously an upper bound of the family with respect to the defined order. This allows for the use of Zorn's lemma, which ensures us of the existence of a maximal element $(A, T)$ in $(\mathcal{F}, \preceq)$. We claim that $A=M$. Assume not and select $x_{0} \in M \backslash A$. Set $\tilde{\mathrm{A}}=A \cup\left\{x_{0}\right\}$ and consider the set

$$
J=\bigcap_{x \in A} B\left(T(x) ; d_{H}\left(T^{*}(x), T^{*}\left(x_{0}\right)\right)\right) \cap T^{*}\left(x_{0}\right) .
$$

Since $T^{*}\left(x_{0}\right)$ is a nonempty closed convex subset of the metric tree $M$, the results of the previous section show that $J \neq \emptyset$ if and only if for each $x \in A$, we have

$$
\operatorname{dist}\left(T(x), T^{*}\left(x_{0}\right)\right) \leq d_{H}\left(T^{*}(x), T^{*}\left(x_{0}\right)\right) .
$$

Using Lemma 3.1 above, we see that $T^{*}\left(x_{0}\right)$ is a proximinal subset of $M$. Therefore the above statement is true if and only if for each $x \in A$,

$$
B\left(T(x), d_{H}\left(T^{*}(x), T^{*}\left(x_{0}\right)\right)\right) \cap T^{*}\left(x_{0}\right) \neq \emptyset .
$$

By the definition of Hausdorff distance, for each $\epsilon>0$ we have

$$
T^{*}(x) \subset N_{d_{H}\left(T^{*}(x), T^{*}\left(x_{0}\right)\right)+\epsilon}\left(T^{*}\left(x_{0}\right)\right) .
$$

However, we are assuming that $T(x) \in T^{*}(x)$, so we must have

$$
B\left(T(x), d_{H}\left(T^{*}(x), T^{*}\left(x_{0}\right)\right)+\epsilon\right) \cap T^{*}\left(x_{0}\right) \neq \emptyset .
$$

Since $T^{*}\left(x_{0}\right)$ is proximinal in $M$ and $\varepsilon$ was arbitrary, we get

$$
B\left(T(x), d_{H}\left(T^{*}(x), T^{*}\left(x_{0}\right)\right)\right) \cap T^{*}\left(x_{0}\right) \neq \emptyset .
$$

Thus, we conclude that $J \neq \emptyset$. Choose $y_{0} \in J$ and define

$$
\tilde{T}(x)= \begin{cases}y_{0} & \text { if } x=x_{0}, \\ T(x) & \text { if } x \in A .\end{cases}
$$

Since

$$
d\left(\tilde{T}\left(x_{0}\right), \tilde{T}(x)\right)=d\left(y_{0}, T(x)\right) \leq d_{H}\left(T^{*}(x), T^{*}\left(x_{0}\right)\right),
$$

for any $x \in A$, we conclude that $\left(A \cup\left\{x_{0}\right\}, \tilde{T}\right) \in \mathcal{F}$, contradicting the maximality of $(A, T)$; therefore $A=M$.

As a direct consequence we get the following Michael-type result.

Corollary 4.1. Let $M$ be a metric tree and $T^{*}: M \rightarrow \mathcal{C}(M)$. Assume $T^{*}$ is continuous; then $T^{*}$ has a continuous selection. Moreover, if $M$ is compact, then $T^{*}$ has a fixed point (i.e., there exists $x \in M$ such that $x \in T^{*}(x)$ ).

The existence of a fixed point is a direct consequence of the main result of [14].

The conclusion of the above theorem will force the selection to inherit some Lipschitz-type condition. Indeed we have the following.

Corollary 4.2. Let $M$ be a metric tree and $T^{*}: M \rightarrow \mathcal{C}(M)$. Assume $T^{*}$ is a Lipschitzian mapping; then $T^{*}$ has a Lipschitzian selection $T: M \rightarrow M$ such that

$$
\operatorname{Lip}(T) \leq \operatorname{Lip}\left(T^{*}\right)
$$

In particular, if the multivalued map $T^{*}$ is nonexpansive, then a nonexpansive selection may be found. This will lead to the following theorem. 
Theorem 4.2. Let $M$ be a bounded metric tree and $T^{*}: M \rightarrow \mathcal{C}(M)$. Assume $T^{*}$ is nonexpansive. Then $T^{*}$ has a fixed point (i.e., there exists $x \in M$ such that $\left.x \in T^{*}(x)\right)$. Moreover, the fixed point set of $T^{*}$ is convex.

Proof. To see that the fixed point set Fix $\left(T^{*}\right)$ is not empty results from the existence of the fixed point of a nonexpansive selection of $T^{*}$. In order to prove that $\operatorname{Fix}\left(T^{*}\right)$ is convex, we need to adapt the proof of the main theorem in this section. Indeed, consider the family $\mathfrak{F}$ of all pairs $(D, T)$, where $\operatorname{Fix}\left(T^{*}\right) \subset D$, and $T: D \rightarrow M, T(d) \in T^{*}(d)$ for all $d \in D, T(x)=x$ for all $x \in F i x\left(T^{*}\right)$, and $d(T(x), T(y)) \leq d(x, y)$ for all $x, y \in D$. By assumption $\left(F i x\left(T^{*}\right), I d\right) \in \mathfrak{F}$, so $\mathfrak{F} \neq \emptyset$. The argument is now a simple modification of the proof of Theorem 4.1. In this case we have

$$
\operatorname{Fix}(T)=\operatorname{Fix}\left(T^{*}\right) .
$$

Since the fixed point set of nonexpansive maps are convex, we get the desired conclusion.

\section{ACKNOWLEDGEMENT}

The authors thank the referee for many useful remarks and for pointing out references [7], [8], and [9].

\section{REFERENCES}

[1] A. G. Aksoy, M. A. Khamsi, Fixed points of uniformly Lipschitzian mappings in metric trees. Preprint.

[2] A. G. Aksoy, B. Maurizi, Metric trees hyperconvex hulls and Extensions. submitted.

[3] N. Aronszajn, P. Panitchpakdi, Extension of uniformly continous transformations and hyperconvex metric spaces. Pacific J. Math. 6 (1956), 405-439. MR0084762 (18:917c)

[4] J. B. Baillon, Nonexpansive mappings and hyperconvex spaces. Contem. Math. 72 (1988), 11-19. MR 0956475 (89k:54068)

[5] I. Bartolini, P. Ciaccia, and M. Patella, String matching with metric trees using approximate distance. SPIR, Lecture Notes in Computer Science, Springer Verlag, Vol. 2476 (2002), 271283.

[6] Y. Benyamini, J. Lindenstrauss, Geometric Nonlinear Functional Analysis, AMS-Colloquium Publications, Vol. 48, 2000. MR.1727673 (2001b:46001)

[7] M. Bestvina, $\mathbb{R}$-trees in topology, geometry, and group theory, Handbook of geometric topology, 55-91, North-Holland, Amsterdam, 2002. MR.1886668 (2003b:20040)

[8] L.M. Blumenthal, Theory and applications of distance geometry, Second Edition, Chelsea Publishing Co., New York, 1970. MR0268781 (42:3678)

[9] M. Bridson, A. Haefliger, Metric spaces of nonpositive curvature, Springer-Verlag, Berlin, Heidelberg, 1999. MR.1744486 (2000k:53038)

[10] P. Buneman, A note on the metric properties of trees. J. Combin. Theory Ser. B, 17 (1974), 48-50. MR0363963 (51:218)

[11] A. W. M. Dress, Trees, tight extensions of metric spaces, and the chomological dimension of certain groups: a note on combinatorial properties of metric spaces. Adv. in Math. 53 (1984), 321-402. MR0753872 (86j:05053)

[12] A. W. M. Dress, V. Moulton and W. Terhalle, T-Theory, An overview. European J. Combin. 17 (1996), 161-175. MR1379369 (97e:05069)

[13] J. R. Isbell, Six theorems about injective metric spaces. Comment. Math. Helv. 39 (1964), 439-447. MR0182949 (32:431)

[14] M. A. Khamsi, KKM and Ky Fan Theorems in Hyperconvex Metric Spaces. J. Math. Anal. Appl. 204 (1996), 298-306. MR 1418536 (98h:54059)

[15] M. A. Khamsi, and W. A. Kirk, "An Introduction to Metric Spaces and Fixed Point Theory". Pure and Applied Math., Wiley, New York, 2001. MR,1818603 (2002b:46002) 
[16] M. A. Khamsi, W. A. Kirk, and C. Marinez-Yañez, Fixed points and selection theorems in hyperconvex spaces. Proc. Amer. Math. Soc. 128, 11 (2000), 3275-3283. MR1777578 (2001g:54048)

[17] M. A. Khamsi, M. Lin, and R. Sine, On the fixed points of commuting nonexpansive maps in hyperconvex spaces, J. Math. Anal. Appl. 168 (1992), 372-380. MR.1175996 (93j:47080)

[18] W. A. Kirk, Hyperconvexity of $\mathbb{R}$-trees. Fund. Math. 156 (1998), 67-72. MR1610559 (98k:54060)

[19] W. A. Kirk, Personal communication.

[20] J. C. Mayer, L. K. Mohler, L. G. Oversteegen, and E. D. Tymchatyn, Characterization of separable metric $\mathbb{R}$-trees. Proc. Amer. Math. Soc. 115, 1 (1992), 257-264. MR1124147 (92h:54049)

[21] J. C. Mayer, L. G. Oversteegen, A Topological Characterization of $\mathbb{R}$-trees. Trans. Amer. Math. Soc. 320, 1 (1990), 395-415. MR0961626 (90k:54031)

[22] J. W. Morgan, ^-trees and their applications. Bull. Amer. Math. Soc. 26 (1992), 87-112. MR1100579 (92e:20017)

[23] F. Rimlinger, Free actions on $\mathbb{R}$-trees. Trans. Amer. Math. Soc. 332 (1992), 313-329. MR1098433 (92j:20021)

[24] C. Semple, and M. Steel, Phylogenetics, Oxford Lecture Series in Mathematics and its Applications, 24, 2003. MR2060009 (2005g:92024)

[25] R. Sine, Hyperconvexity and nonexpansive multifunctions. Trans. Amer. Math. Soc. 315 (1989), 755-767. MR0954603 (90a:54054)

[26] J. Tits, A Theorem of Lie-Kolchin for Trees. Contributions to algebra: a collection of papers dedicated to Ellis Kolchin, Academic Press, New York, 1977. MR 0578488 (58:28205)

[27] M. Zippin, Application of Michael's continous selection theorem to operator extension problems. Proc. Amer. Math. Soc. 127, 5 (1999), 1371-1378. MR1487350 (99h:46040)

Department of Mathematics, Claremont McKenna College, Claremont, California 91711

E-mail address: asuman.aksoy@claremontmckenna.edu

Department of Mathematical Sciences, University of Texas at El Paso, El Paso, TEXAS 79968-0514

E-mail address: mohamed@math.utep.edu 